\title{
Three dimensional vibration analysis of a class of traction-free solid elastic bodies with an eccentric cavity
}

\author{
Seyyed M. Hasheminejad ${ }^{\mathrm{a}, *}$ and Yaser Mirzaei ${ }^{\mathrm{b}}$ \\ ${ }^{a}$ Acoustics Research Laboratory, Department of Mechanical Engineering, Iran University of Science and \\ Technology, Narmak, Tehran, Iran \\ ${ }^{\mathrm{b}}$ Department of Mechanical Engineering, Damavand Branch, Islamic Azad University, Damavand, Iran
}

Received 4 July 2011

Revised 8 January 2012

\begin{abstract}
A three-dimensional elasticity-based continuum model is developed for describing the free vibrational characteristics of an important class of isotropic, homogeneous, and completely free structural bodies (i.e., finite cylinders, solid spheres, and rectangular parallelepipeds) containing an arbitrarily located simple inhomogeneity in form of a spherical or cylindrical defect. The solution method uses Ritz minimization procedure with triplicate series of orthogonal Chebyshev polynomials as the trial functions to approximate the displacement components in the associated elastic domains, and eventually arrive at the governing eigenvalue equations. An extensive review of the literature spanning over the past three decades is also given herein regarding the free vibration analysis of elastic structures using Ritz approach. Accuracy of the implemented approach is established through proper convergence studies, while the validity of results is demonstrated with the aid of a commercial FEM software, and whenever possible, by comparison with other published data. Numerical results are provided and discussed for the first few clusters of eigen-frequencies corresponding to various mode categories in a wide range of cavity eccentricities. Also, the corresponding 3D mode shapes are graphically illustrated for selected eccentricities. The numerical results disclose the vital influence of inner cavity eccentricity on the vibrational characteristics of the voided elastic structures. In particular, the activation of degenerate frequency splitting and incidence of internal/external mode crossings are confirmed and discussed. Most of the results reported herein are believed to be new to the existing literature and may serve as benchmark data for future developments in computational techniques.
\end{abstract}

Keywords: Elastic structure, eccentric void, free-oscillations, multiple degeneracies, modal splitting, mode cross-over

\section{Introduction}

The rapid advancement in contemporary industries towards high precision in engineering applications calls for more accurate predictions of the dynamic behavior of mechanical systems. Accordingly, as computers and analytical procedures become more efficient, the three dimensional vibrationanalysisof structural elements of various shapes based on the small-strain linear elasticity theory has received increasing attention in the recent decades. Such studydoes not rely on any hypotheses involving the kinematics of deformation and providesaccurate realistic resultswhich cannot otherwise be predicted by approximate theories. Unfortunately, exact three-dimensional elastodynamic solutions are accessible only for simple (canonical) geometries and boundary conditions (e.g., solid or hollow spheres [1]

\footnotetext{
* Corresponding author: Seyyed M. Hasheminejad, Acoustics Research Laboratory, Department of Mechanical Engineering, Iran University of Science and Technology, Narmak, Tehran 16846-13114, Iran. Tel.: 98912 7371354; Fax: 9821 77240488; E-mail: hashemi@iust.ac.ir.
} 
and cylinders [2]). Consequently, many approximate methods such as the finite element method [3], the boundary element method [4], and the Ritz method [5-7] have been developed over the years. Among these methods, the Ritz continuum approachhas been demonstrated effective and shows special advantages such as high accuracy, small computational cost, and easy coding. It has evolved steadily over the years with the development of more efficient and accurate global admissible functions. Its capability to accommodate a wider spectrum of elastic domain configurations, boundary constraints and loading conditions has also improved and further enhanced its applications in many interesting engineering situations. The following three paragraphs contain brief overviews on the three major variants of Ritz solution procedure which respectively use simple algebraic polynomials (firstly used by Leissa and coworkers [8-10]), generated orthogonal polynomials (firstly used by Liew and coworkers [11-14]), and Chebyshev polynomials (firstly used by Zhou and coworkers [15-18]) as admissible functions to study free vibrations of elastic bodies of various shapes based on the three dimensional linear elasticity theory.

Simple algebraic polynomials were used as admissible functions in the 3D Ritz method in conjunction with the three dimensional linear elasticity theoryto study free vibrationsof homogeneous isotropicrectangular cantilevered parallelepipeds [19], twisted parallelepipeds [20], a truncated quadrangular pyramid with a clamped base [21], solids of fairly general shape (a truncated triangular prism, a truncated cone, a rectangular parallelepiped with a rectangular cut-out and a fin-shaped solid) [22], cantilevered skew plates [23], solids of revolution [24], thick-walled closed spherical bodies of revolution [25], cantilevered solid isotropic cylinders [8], completely free hollow cylinders [9], free circular and annular plates [10], thick spherical shell segments of varying thickness [26], solids of revolution (circular disks, conical/cylindrical/spherical shells) [27], thick nonlinearly tapered annular and circular plates [28], solid cones with and without an axial circular cylindrical hole [29], solid and hollow hemi-ellipsoids of revolution with and without an axial circular cylindrical hole [30], solid paraboloids and complete paraboloidal shells of revolution with variable wall thickness [31], and just recently, cylindrical elastic solids and thick circular plates and cylinders with V-notches and sharp radial cracks [32].

Generated orthogonal polynomials were also used as trial functions in the 3D Ritz method to study threedimensional free vibrations of homogeneous isotropic circular and annular plates with various boundary conditions [11,12], thick rectangular plates with arbitrary combinations of boundary constraints [33], skewed trapezoidal plates of different planforms [34], elliptic bars with various end constraints [13], hollow cantilevered cylinders with an arbitrary cross section [35], solid and hollow cylinders of arbitrary cross section with different end conditions [14], thick and open cylindrical shells [36], and more recently, rectangular plates of arbitrary thickness and boundary conditions [37].

High accuracy, stable numerical computation, and rapid convergence have recently been observed through adoption of Chebyshev polynomials as global admissible functions in the 3D Ritz method based on the three-dimensional elasticity theory. In particular, Zhou and coworkers employed the Chebyshev-Ritz procedure to study three dimensional free vibration characteristics of arbitrary thick rectangular plates with various uniform boundary conditions [38], a torus with a circular cross section [39], circular and annular plates with any boundary conditions [40], solid and hollow circular cylinders [18], rectangular thick plates resting on elastic Pasternak foundations [41], generalized super-elliptical plates [42], cantilevered thick skew plates [43], and just recently, circular toroidal sectors with circular cross-section [5].

When structural abnormalities like manufacturing tolerances, geometric variations, and material inhomogeneities are present or an eccentric void, inclusion, or cut-out is brought in, some of the degenerate natural frequencies which are coincident in the perfectly symmetric case divide into distinct values, mode shapes become distorted, and beating among intimately spaced vibration modesmay be produced $[1,2,44]$. To circumvent this situation, the vibration characteristics of the axisymmetric structures with asymmetric features should be investigated carefully. Several authors have analyzed the impact of different types of geometric asymmetries or imperfections on the vibrational behavior of nominally axi-symmetric structures. Specifically, quantitative assessment of the frequency splitting and modal shape deformation in non-uniform rings and shells, as well as eccentric discs, plates, cylinders, and spheres has been performed. Among them, Yu and Mote [45] studied the effects of radial slots of circular plates with rotating load and provided a rule for natural frequency splitting in circular plates with equally spaced identical radial slots. Chen and Zhou [46] illustrated the low-frequency mode shapes of a small disc with eccentric holes based on the boundary element approach. Shen and Mote [47] presented the sufficient conditions governing mode splitting in a two-dimensional,degenerate, mechanical system whose eigen-solutions satisfy the Helmholtz equation in square and 
(a)

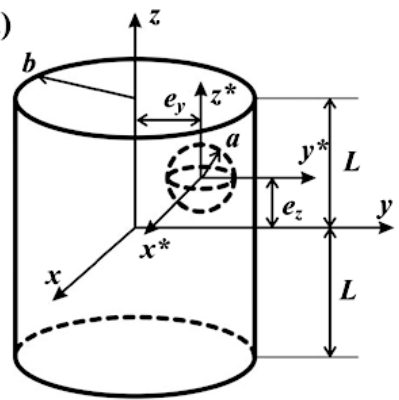

(c)

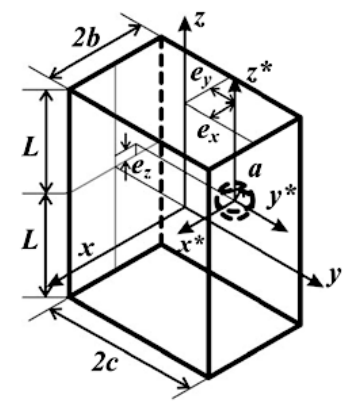

(b)

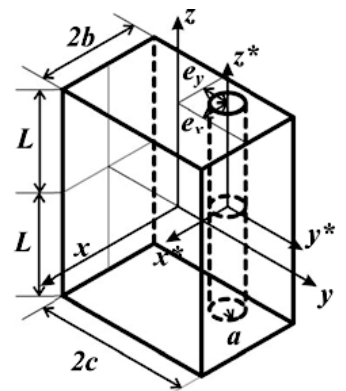

(d)

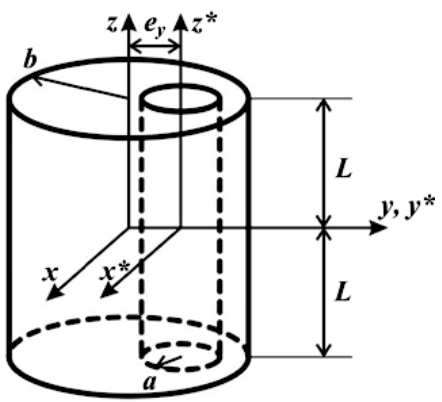

(e)

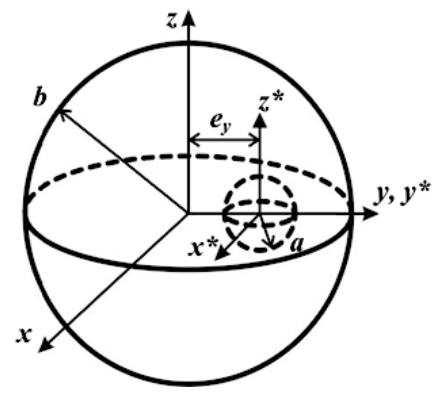

Fig. 1. Problem geometries. (a) finite cylinder with an internal spherical cavity. (b) solid parallelepiped with an internal cylindrical cavity. (c) solid parallelepiped with an internal spherical cavity. (d) finite cylinder with an internal cylindrical cavity. (e) solid sphere with an internal spherical cavity.

circulardomains with cracks. Tseng and Wickert [48] studied the vibration of an eccentrically clamped annular plate and pointed out splitting of the degenerate natural frequencies. Parker and Mote [49-51] used perturbation analysis to investigate the eigen-solutions for plate vibration and the wave equation on annular domains with boundary shape or stiffness variations, and discussed the phenomenon of natural frequency splitting. Kim et al. [44] presented a natural frequency splitting rule for general rotationally periodic structures and investigated the effects of imperfection on both repeated and split natural frequency modes. Chang and Wickert [52] studied the vibration of rotationally periodic structures and obtained a natural frequency splitting rule and a mode contamination rule for axisymmetric structures with identical, evenly spaced asymmetries. Hasheminejad and Mirzaei [1,2] employed the appropriate translational addition theorems to find exact 3D elasticity solutions for free vibrations of eccentric circular cylinders and eccentric spheres.

The above review clearly indicates that the effects of wall thickness non-uniformity or internal hole/cutout eccentricity on vibrational characteristics of various thin-walled structures have been thoroughly investigated. Also, except in Refs. [1,2] where the exact three-dimensional elastodynamic solutions are provided for eccentric hollow spheres and cylinders, rigorous analytic, semi-analytic, or numerical studieson free vibrations ofunrestrained elastic bodiesof canonical shape (e.g., spheres, cylinders, parallelepipeds)containing an eccentric internal cavity appears tobe almost nonexistent orvery sparse. Accordingly, the main purpose of the current work is to employ the Chebyshev-Ritz solution procedure in conjunction with the three-dimensional linear elasticity theory to fill this gap (see Fig. 1). It is noteworthy that due to the excellent properties of Chebyshev polynomials in numerical operations, 
the adopted method is well known to predict more frequencies and modes with higher convergence rate, better numerical stability, and improved accuracy in comparison with other types of admissible functions such as simple algebraic polynomials, particularly in the 3-D vibration analysis of an elastic bodies where numerical instability may occur with a great number of terms of admissible functions [38,41,53]. This fact was underlined by Zhou et al. [40] where they demonstrated that by using Chebyshev polynomials instead of simple polynomials as the admissible functions, the immunity against ill-conditioned behavior in computing eigenfrequencies of completely free solid and annular thick circular plates can be greatly enhanced. The presented 3D solutionscanform a helpful guide for design engineers in evaluating the influence of changingthe cavity eccentricity on the spectral response of suchstructural componentsin various technological applications [53-59]. It can specifically accompany experimental procedures for identification/characterization of internal structural non-uniformities [60-66]. Lastly, the computed complete spectrum of eigen-frequencies and mode shapes can reveal the physical characteristics of the problem and also serve as the benchmark for assessment of other numerical or asymptotic solutions.

\section{Formulation}

\subsection{Basic field equations}

Consider the group of linear, macroscopically homogeneous, isotropic, and traction-free canonically shaped elastic bodies depicted in Fig. 1, as described below: Fig. 1a (finite cylinder with an eccentric spherical cavity), Fig. 1b (rectangular parallelepiped with an eccentric cylindrical cavity), Fig. 1c (rectangular parallelepiped with an eccentric spherical cavity), Fig. 1d (finite cylinder with an eccentric cylindrical cavity), and Fig. 1e (solid sphere with an eccentric spherical cavity). The problem geometry in each case is initially described in terms of the pair of Cartesian coordinates, $(x, y, z)$ and $\left(x^{*}, y^{*}, z^{*}\right)$, the origins of which are positioned at the centers of the elastic body and the eccentric cavity, respectively. Also, the corresponding displacement components at a generic point within the body are denoted by $u, v$ and $w$ in the $x, y$ and $z$ directions, respectively. The analysis procedure is based on the exact, small-strain, three-dimensional linear elasticity theory. The elastic strain energy, $V$, and the kinetic energy, $T$, of each elastic body undergoing a small amplitude vibration are given by the volume integrals

$$
\begin{aligned}
& T=\frac{\rho}{2} \iiint\left(\dot{u}^{2}+\dot{v}^{2}+\dot{w}^{2}\right) d x d y d z \\
& V=\frac{1}{2} \iiint \sigma_{i j} \varepsilon_{i j} d x d y d z
\end{aligned}
$$

where $\rho$ is the solid material density, and $(\dot{u}, \dot{v}, \dot{w})$ denote the components of the velocity vector, and $\sigma_{i j}$ and $\varepsilon_{i j}$ are the stress and strain tensors, respectively. Also, the generalized Hooke's law is written as

$$
\sigma_{i j}=\lambda \delta_{i j} \varepsilon_{k k}+2 G \varepsilon_{i j}
$$

where $\delta_{i j}$ is the Kronecker delta symbol, $\left(\lambda=\frac{\nu E}{(1+\nu)(1-2 \nu)}, \mu=\frac{E}{2(1+\nu)}\right)$ are Lame constants, $E$ is Young's modulus, and $\nu$ is Poisson's ratio. By direct substitution of Eq. (2) into Eq. (1), the potential energy is written in the form

$$
V=\frac{E}{2(1+\nu)} \iiint\left(\frac{\nu}{1-2 \nu} \Lambda_{1}+\frac{1}{2} \Lambda_{2}+\Lambda_{3}\right) d x d y d z,
$$

where $\Lambda_{1}=\left(\varepsilon_{x x}+\varepsilon_{y y}+\varepsilon_{z z}\right)^{2}, \Lambda_{2}=\left(\varepsilon_{x y}^{2}+\varepsilon_{x z}^{2}+\varepsilon_{y z}^{2}\right), \Lambda_{3}=\left(\varepsilon_{x x}^{2}+\varepsilon_{y y}^{2}+\varepsilon_{z z}^{2}\right)$, in which the strain components for small deformations in the Cartesian coordinates are written as

$$
\begin{aligned}
& \varepsilon_{x x}=\frac{\partial u}{\partial x}, \quad \varepsilon_{y y}=\frac{\partial v}{\partial y}, \quad \varepsilon_{z z}=\frac{\partial w}{\partial z} \\
& \varepsilon_{x y}=\frac{\partial u}{\partial y}+\frac{\partial v}{\partial x}, \quad \varepsilon_{x z}=\frac{\partial u}{\partial z}+\frac{\partial w}{\partial x}, \quad \varepsilon_{y z}=\frac{\partial v}{\partial z}+\frac{\partial w}{\partial y},
\end{aligned}
$$


Table 1

The convergence rates of the first three natural frequencies ofthe considered class of traction-free elastic bodies for selected geometric parameters

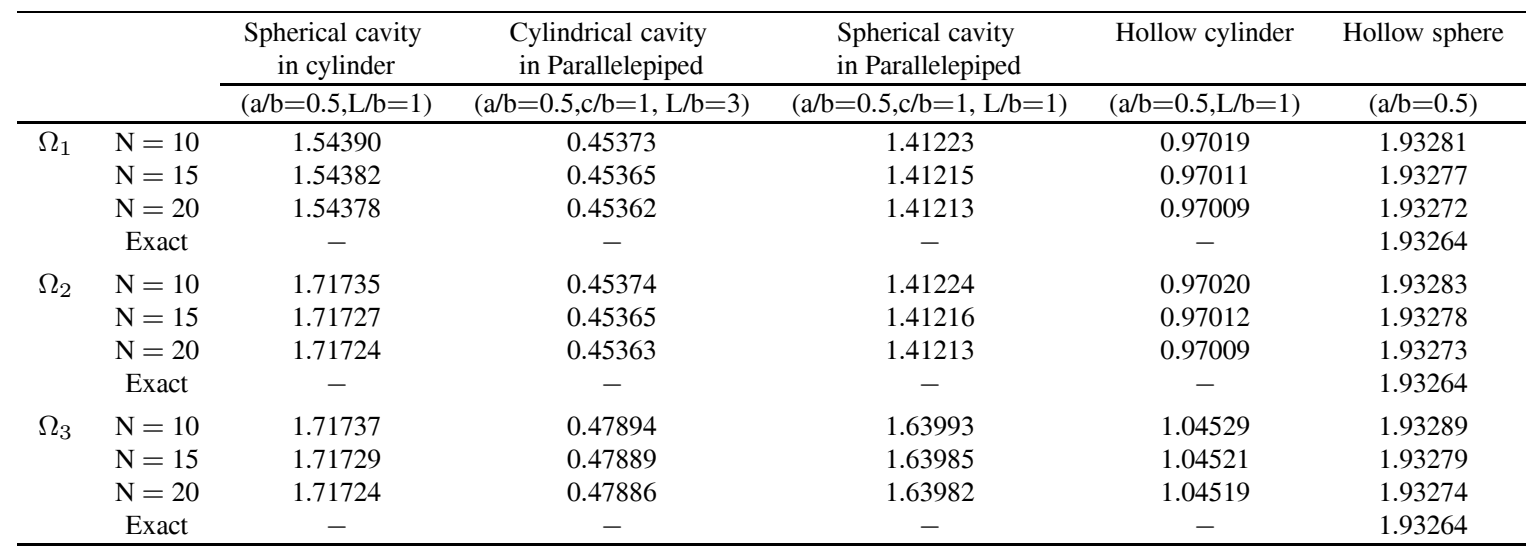

For free vibration analysis, simple harmonic motion is generally assumed. Accordingly, the displacement components may be expressed as

$$
u(x, y, z, t)=U(x, y, z) e^{\mathrm{i} \omega t}, v(x, y, z, t)=V(x, y, z) e^{\mathrm{i} \omega t}, w(x, y, z, t)=W(x, y, z) e^{\mathrm{i} \omega t},
$$

where $\omega$ denotes the natural frequency, $\mathrm{i}=\sqrt{-1}$, and $(U, V, W)$ are displacement amplitude functions, which may advantageously be represented by triplicate series of Chebyshev polynomial trial functions for traction-free elastic bodies in the form [5]

$$
\begin{aligned}
& U(x, y, z)=\sum_{i=1}^{I} \sum_{j=1}^{J} \sum_{k=1}^{K} A_{i j k} F_{i}(x) F_{j}(y) F_{k}(z), \\
& V(x, y, z)=\sum_{i=1}^{L} \sum_{j=1}^{M} \sum_{k=1}^{N} B_{i j k} F_{i}(x) F_{j}(y) F_{k}(z), \\
& W(x, y, z)=\sum_{i=1}^{P} \sum_{j=1}^{Q} \sum_{k=1}^{H} C_{i j k} F_{i}(x) F_{j}(y) F_{k}(z),
\end{aligned}
$$

where the integers $I$ through $H$ are truncation constants, $\left(A_{i j k}, B_{i j k}, C_{i j k}\right)$ are unknown coefficients, and the Chebyshev polynomials are generally defined in terms of cosine functions as $F_{j}(\xi)=[\cos (j-1) \arccos (\xi)](j=$ $1,2,3, \ldots)[5]$. Here, it should be noted that each of the displacement amplitude functions $(U, V, W)$ is generally written in the form of a triplicate series of Chebyshev polynomials multiplied by a boundary function which ensures that the displacement components satisfy the essential geometric boundary conditions. Keeping in mind the unconstrained (stress-free) boundary conditions on the cavity surface and also on the outer surface of the elastic body, these boundary functions are naturally assumed to be unity (e.g., see Table 1 in Ref. [53]).

The Ritz procedure requires a minimization of the Lagrangian functional $\Pi=T_{\max }-V_{\max }$, where $T_{\max }$ is the maximum kinetic energy and $V_{\max }$ is the maximum strain energy, written as

$$
\begin{aligned}
& T_{\max }=\frac{\rho \omega^{2}}{2} \iiint\left[U^{2}(x, y, z)+V^{2}(x, y, z)+W^{2}(x, y, z)\right] d x d y d z \\
& V_{\max }=\frac{E}{2(1+\nu)} \iiint\left[\frac{\nu}{1-2 \nu} \Gamma_{1}(x, y, z)+\frac{1}{2} \Gamma_{2}(x, y, z)+\Gamma_{3}(x, y, z)\right] d x d y d z,
\end{aligned}
$$

where the $\Lambda_{j}(x, y, z, t)=\Gamma_{j}(x, y, z) e^{\mathrm{i} \omega t}(j=1,2,3)$ assumption has been employed. Substitution of Chebyshev expansions (6) into the above two equations, going through the volume integrations over the entire physical domain for each elastic body as outlined in the Appendix (see Fig. 1), and subsequent minimization of the functional $\Pi$ 
with respect to the unknown coefficients (i.e., $\frac{\partial \Pi}{\partial A_{i j k}}=\frac{\partial \Pi}{\partial B_{i j k}}=\frac{\partial \Pi}{\partial C_{i j k}}=0$ ), leads to the following general linear eigenvalue problem for each elastic body

$$
\left[\mathbf{K}-\omega^{2} \mathbf{M}\right]\left[\begin{array}{l}
\mathbf{A} \\
\mathbf{B} \\
\mathbf{C}
\end{array}\right]=0,
$$

where the column vectors $\mathbf{A}, \mathbf{B}$ and $\mathbf{C}$, contain the unknown coefficients, and are given as

$$
\begin{aligned}
& \mathbf{A}=\left\{A_{111}, A_{112}, \ldots, A_{11 K} ; A_{121}, A_{122}, \ldots, A_{12 K} ; \ldots ; A_{1 J 1}, A_{1 J 2}, \ldots, A_{1 J K} ; \ldots ; A_{i I J 1}, A_{i I J 2}, \ldots, A_{I J K}\right\}^{T}, \\
& \mathbf{B}=\left\{B_{111}, B_{112}, \ldots, B_{11 N} ; B_{121}, B_{122}, \ldots, B_{12 N} ; \ldots ; B_{1 M 1}, B_{1 M 2}, \ldots, B_{1 M N} ; \ldots ; B_{L M 1}, B_{L M 2},\right. \\
&\left.\ldots, B_{L M N}\right\}^{T}, \\
& \mathbf{C}=\left\{C_{111}, C_{112}, \ldots, C_{11 H} ; C_{121}, C_{122}, \ldots, C_{12 H} ; \ldots ; C_{1 Q 1}, C_{1 Q 2}, \ldots, C_{1 Q H} ; \ldots ; C_{P Q 1}, C_{P Q 2}, \ldots, C_{P Q H}\right\}^{T} .
\end{aligned}
$$

Also, $\mathbf{K}$ and $\mathbf{M}$ are stiffness and mass matrices resulting from minimization of the maximum strain and kinetic energies, respectively. Here, it should be noted that since the Chebyshev polynomial function series described by expansions (6) form a mathematically complete set of functions, the results obtained from the Ritz minimization process will converge monotonically from above to the exact frequencies as the number of terms in each series tends to infinity [10]. Also, non-trivial solutions of the eigen-system (8) can be obtained by setting the determinant of the coefficient matrix equal to zero (i.e., $\left|\mathbf{K}-\omega^{2} \mathbf{M}\right|=0$ ). The roots of the determinant are the squares of the eigen-values or eigen-frequencies, which are the upper bounds on the exact frequency values. The associated eigen-functions or the mode shapes, are determined by subsequent back-substitution of the individual eigenvalues into the set of algebraic Eq. (8), and solving for the ratios of unknown coefficients through implementation of numerical methods such as the QR algorithm. In the next section, we consider a number of numerical examples in order to illustrate the nature and general behavior of solution.

\section{Numerical results}

As is well known, the Ritz method can provide accurate solutions. However, its efficiency depends greatly on the choice of admissible functions. The natural frequencies obtained by the Ritz method converge as upper bounds to the exact values. These upper bound estimates converge to the exact value by increasing the number of terms of admissible functions in the computation and hence solution of any accuracy can be obtained in theory. However, a practical limit to the number of terms used always exists because of the limited speed, the capacity and the numerical accuracy of computers. In the 3D vibration analysis of an elastic body in particular, numerical instability may occur with a great number of terms of admissible functions, especially when triplicate series are used. Therefore, the validity of a numerical method often hinges on the convergence rate, the numerical stability and the accuracy of the method.

Chebyshev polynomial series is a set of complete and orthogonal series in the interval $[-1,1]$. This ensures that the triple series is also a complete and orthogonal set in the region. Moreover, excellent properties of Chebyshev polynomial series in the approximation of functions have been well known. Therefore, more rapid convergence and better stability in numerical operation than other polynomial series such as the simple algebraic polynomials and the generated orthogonal polynomials can be expected. Table 1 gives the convergence rate of the first three normalized natural frequencies $(\Omega=b \omega \sqrt{\rho / \mu})$ for selected dimensionless geometric parameters for all subproblems displayed in Fig. 1, in the absence of cavity eccentricity. It is clear that the convergence rate is very rapid in all cases. Also, by increasing the number of Chebyshev polynomial functions, the frequency parameters monotonously decrease and approach the exact values from above with minimal computational difficulties (e.g., see the last column in Table 1 in which the exact values are taken from Ref. [1]). Also, by adopting truncation constants of $I=J=K=L=M=N=P=Q=H=20$, the adequate convergence of all calculated natural frequencies to at least three-digit exactitude can be assured. Here it should be noted that, for the sake of simplicity, equal numbers of the Chebyshev polynomial terms were used for the displacement components in their respective 

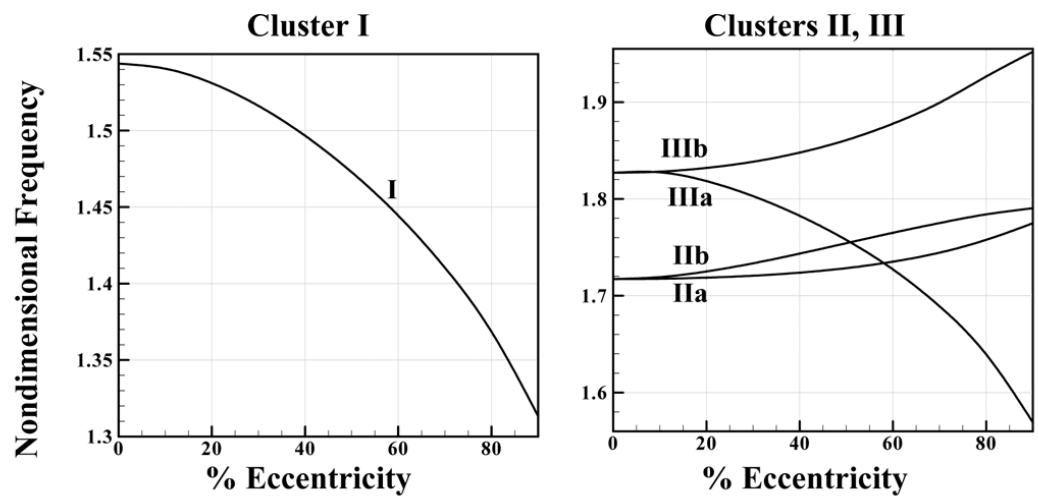

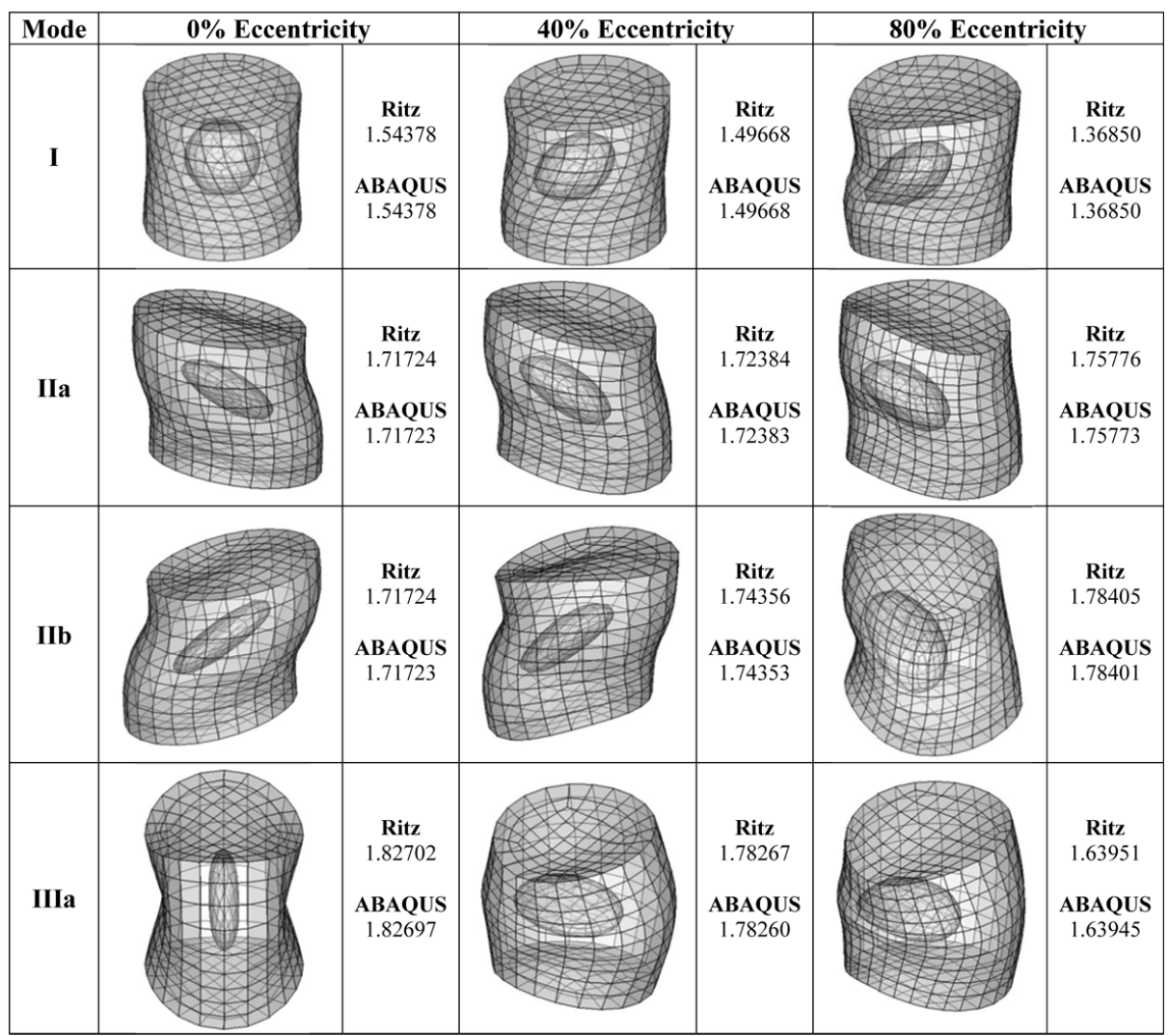

Fig. 2. Variation of the first three frequency clusters with eccentricity parameter for the finite cylinder with an internal spherical cavity, along with the first four three-dimensional mode shapes for selected eccentricity parameters.

coordinate directions. Nevertheless, in many cases, using unequal numbers of series terms may be more efficient. Lastly, the convergence patterns for the eccentric sub-problems were found to be very similar to those presented in Table 1 (results are not tabulated for briefness).

A Mathematica code [67] was constructed for numerical treatment of the eigen-system (8) for each sub-problem, i.e., to calculate the resonance frequencies and to determine the unknown coefficients (mode shapes) as a function of the inner cavity eccentricity. The convergence of numerical solutions was systematically checked in a simple trial and error manner, by increasing the truncation constant (i.e., including higher number of terms) while looking for steadiness or stability in the numerical value of the solutions, as explained above (see Table 1). Figures 2 through 6 display the variation of the first few clusters of dimensionless natural frequencies, $\Omega$, with the eccentricity parameter $\left(0 \leqslant \bar{e}=e_{y} / b \leqslant 0.9 ; e_{x}=e_{z}=0\right)$ for the sub-problems displayed in Fig. 1 with the following selected 

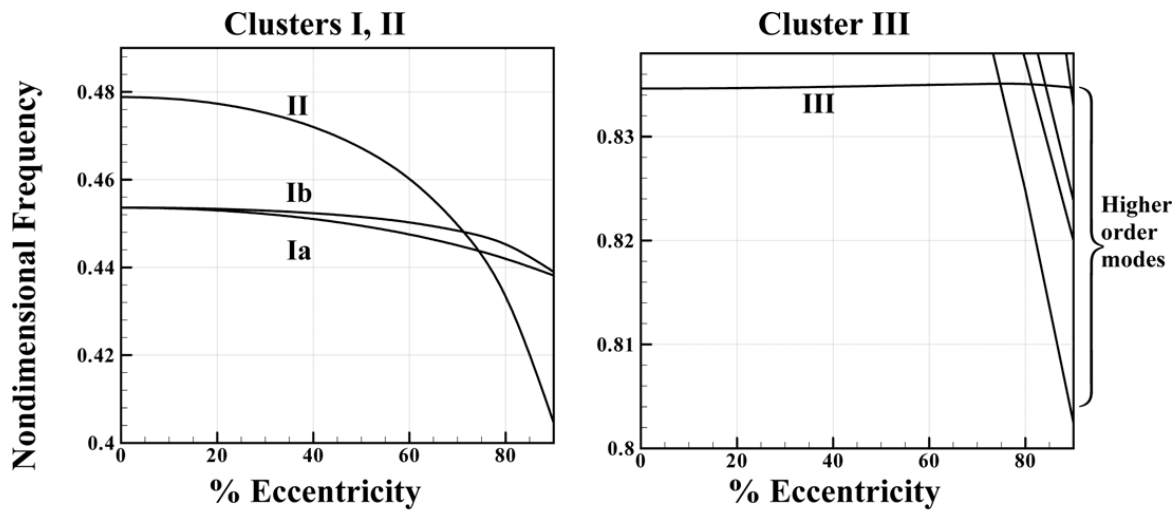

\begin{tabular}{|c|c|c|c|}
\hline Mode & $0 \%$ Eccentricity & $40 \%$ Eccentricity & $80 \%$ Eccentricity \\
\hline Ia & $\begin{array}{c}\text { Ritz } \\
0.45362 \\
\\
\text { ABAQUS } \\
0.45362\end{array}$ & $\begin{array}{c}\text { Ritz } \\
0.45103 \\
\text { ABAQUS } \\
0.45103\end{array}$ & $\begin{array}{c}\text { Ritz } \\
0.44197 \\
\text { ABAQUS } \\
0.44197\end{array}$ \\
\hline Ib & $\begin{array}{c}\text { Ritz } \\
0.45363 \\
\\
\text { ABAQUS } \\
0.45362\end{array}$ & $\begin{array}{c}\text { Ritz } \\
0.45238 \\
\text { ABAQUS } \\
0.45236\end{array}$ & $\begin{array}{c}\text { Ritz } \\
0.44527 \\
\text { ABAQUS } \\
0.44526\end{array}$ \\
\hline II & $\begin{array}{c}\text { Ritz } \\
0.47886 \\
\text { ABAQUS } \\
0.47884\end{array}$ & $\begin{array}{c}\text { Ritz } \\
0.47205 \\
\text { ABAQUS } \\
0.47200\end{array}$ & $\begin{array}{c}\text { Ritz } \\
0.43342 \\
\text { ABAQUS } \\
0.43338\end{array}$ \\
\hline III & $\begin{array}{c}\text { Ritz } \\
0.83465 \\
\text { ABAQUS } \\
0.83462\end{array}$ & $\begin{array}{c}\text { Ritz } \\
0.83485 \\
\text { ABAQUS } \\
0.83479\end{array}$ & $\begin{array}{c}\text { Ritz } \\
0.83509 \\
\text { ABAQUS } \\
0.83505\end{array}$ \\
\hline
\end{tabular}

Fig. 3. Variation of the first three frequency clusters with eccentricity parameter for the solid parallelepiped with an internal cylindrical cavity, along with the first four three-dimensional mode shapes for selected eccentricity parameters.

dimensionless geometric parameters; Fig. 2: $L / b=1, a / b=0.5$; Fig. 3: $L / b=3, a / b=0.5, c / b=1$; Fig. 4 : $a / b=0.5, c / b=1, L / b=1$; Fig. 5: $L / b=1, a / b=0.5$; Fig. $6: a / b=0.5$. The first four 3D deformed mode shapes are also shown in each figure for selected eccentricity parameters $(\bar{e}=0,0.4,0.8)$. Moreover, the calculated dimensionless frequencies are compared with the corresponding values computed using the finite element code ABAQUS [68]. It is clear that excellent agreements are obtained for all eccentricities in each sub-problem. In these validations, normally about 20,000 twenty-noded brick elements (C3D20R) were used to model each eccentric elastic body. Also shown are the excellent agreements found with the only available exact results in the open literature [1], which corresponds to the free vibration characteristics of the eccentric elastic sphere (Fig. 1e). The most important observations are summarized as follows. 

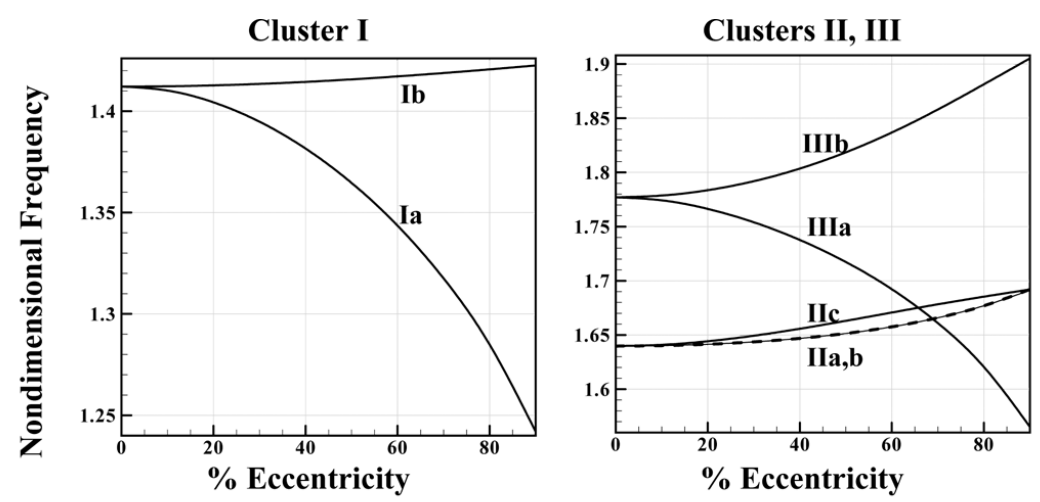

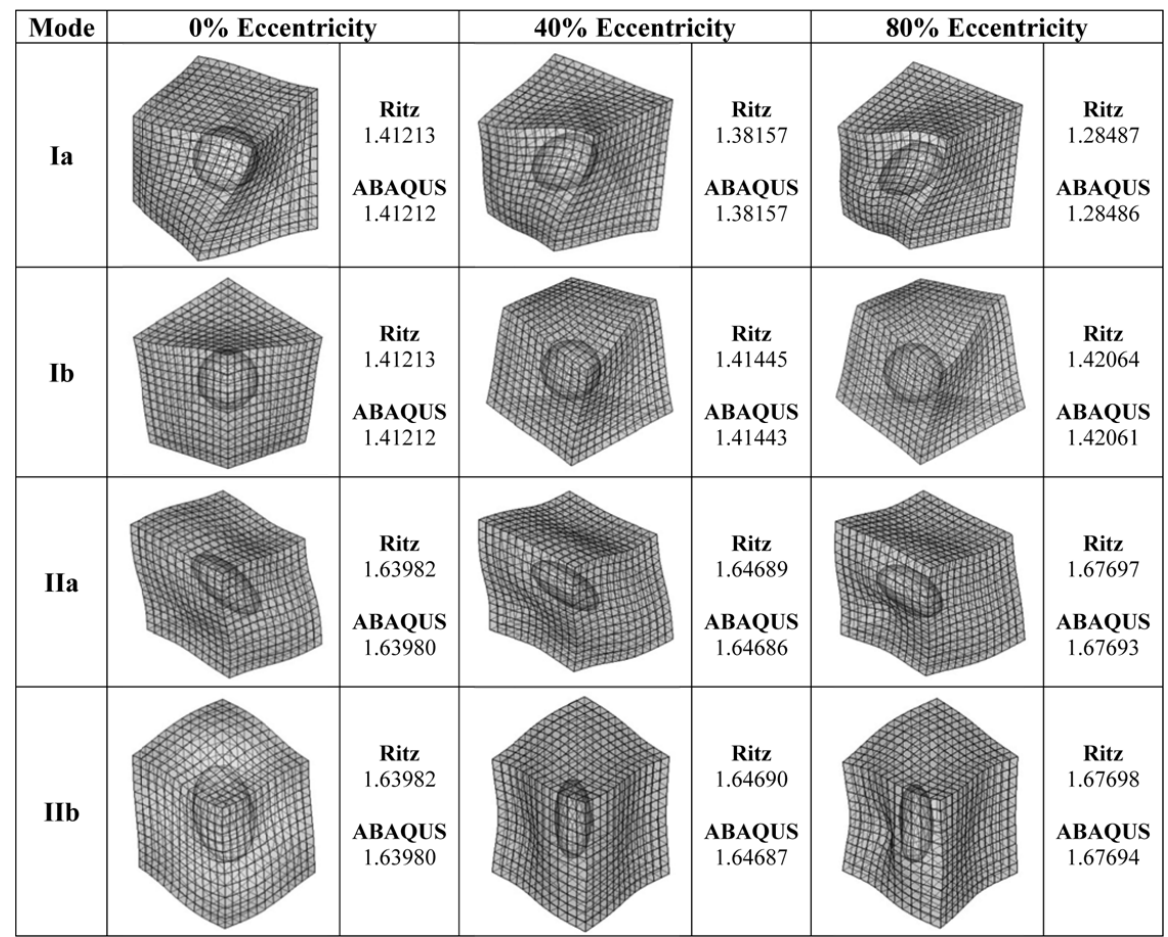

Fig. 4. Variation of the first three frequency clusters with eccentricity parameter for the solid parallelepiped with an internal spherical cavity, along with the first four three-dimensional mode shapes for selected eccentricity parameters.

The modal spectrums of elastic solids exhibit very unique characteristics. In the absence of eccentricity $(\bar{e}=0)$, due to the presence of geometric symmetries, perfectly repeated fully degenerate (multiplet) frequencies are observed. For example, one may consider the first frequency cluster (i.e., cluster I) associated with the spheroidal modes for the concentric elastic sphere (i.e., see the first column of Fig. 6; also see [1]), for which there is a total of five fully degenerate frequencies (note that only the first four mode shapes corresponding to sub-clusters Ia,b,c,d are displayed, due to the space limit). Similarly, multiple degeneracies are observed for clusters II and III in Fig. 2, cluster I in Fig. 3, clusters II and III in Fig. 4, clusters I and II in Fig. 5, and clusters I and II in Fig. 6. Also, a more careful examination of mode shapes presented in the first column of each figure demonstrates that, due to problem symmetry, certain mode shapes associated with the degenerate frequency clusters occur in pairs (i.e., they are exactly the same, but merely rotated). In particular, the degenerate mode pairs IIa,b and IIIa,b are observed in Fig. 2, modes Ia,b in Fig. 3, modes Ia,b, IIa,b and IIIa,b in Fig. 4, modesIa,b and IIa,b in Fig. 5, and modes Ia,b, Ic,d, IIa,b, and IIc,d in Fig. 6. 

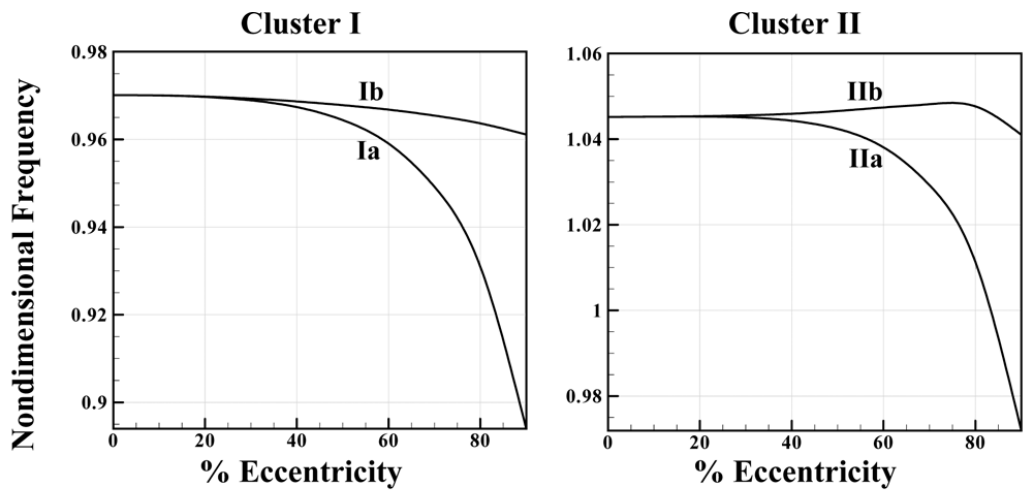

\begin{tabular}{|c|c|c|c|c|}
\hline Mode & 0\% Eccentricity & $40 \%$ Eccentricity & $80 \%$ Ecce & icity \\
\hline Ia & $\begin{array}{c}\text { Ritz } \\
0.97009 \\
\\
\text { ABAQUS } \\
0.97008\end{array}$ & $\begin{array}{c}\text { Ritz } \\
0.96737 \\
\\
\text { ABAQUS } \\
0.96736\end{array}$ & 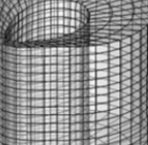 & $\begin{array}{c}\text { Ritz } \\
0.93096 \\
\\
\text { ABAQUS } \\
0.93096\end{array}$ \\
\hline Ib & $\begin{array}{c}\text { Ritz } \\
0.97009 \\
\\
\text { ABAQUS } \\
0.97008\end{array}$ & $\begin{array}{c}\text { Ritz } \\
0.96868 \\
\\
\text { ABAQUS } \\
0.96866\end{array}$ & & $\begin{array}{c}\text { Ritz } \\
0.96367 \\
\\
\text { ABAQUS } \\
0.96365\end{array}$ \\
\hline IIa & $\begin{array}{c}\text { Ritz } \\
1.04519 \\
\\
\text { ABAQUS } \\
1.04517\end{array}$ & $\begin{array}{c}\text { Ritz } \\
1.04435 \\
\text { ABAQUS } \\
1.04431\end{array}$ & & $\begin{array}{c}\text { Ritz } \\
1.01136 \\
\text { ABAQUS } \\
1.01134\end{array}$ \\
\hline IIb & $\begin{array}{c}\text { Ritz } \\
1.04522 \\
\\
\text { ABAQUS } \\
1.04517\end{array}$ & $\begin{array}{c}\text { Ritz } \\
1.04597 \\
\\
\text { ABAQUS } \\
1.04593\end{array}$ & 梅 & $\begin{array}{c}\text { Ritz } \\
1.04771 \\
\\
\text { ABAQUS } \\
1.04767\end{array}$ \\
\hline
\end{tabular}

Fig. 5. Variation of the first two frequency clusters with eccentricity parameter for the finite cylinder with an internal cylindrical cavity, along with the first four three-dimensional mode shapes for selected eccentricity parameters.

As eccentricity is introduced, characteristically different effects on natural frequencies are observed, depending on the problem geometry and mode number. In particular, none of the frequency clusters remain fully degenerate, and the imposed asymmetry causes some or all of the repeated vibration modes to split into modes with distinct natural frequencies. More specifically, it is observed that clusters (II, III) are two-fold degenerate and split into sub-clusters (IIa,b; IIIa,b) in Fig. 2, cluster I is two-fold degenerate and split into sub-clusters Ia,b in Fig. 3, clusters (I, III) are two-fold degenerate and split into sub-clusters (Ia,b; IIIa,b) while cluster II is three-fold degenerate and splits into two-fold degenerate sub-clusters IIa,b in addition to an isolated sub-cluster IIc in Fig. 4, clusters (I, II) are two-fold degenerate and split into sub-clusters (Ia,b; IIa,b) in Fig. 5, clusters (I, II) are five-fold degenerate and each split into two distinct two-fold degenerate sub-clusters (Ia,b; Ic,d; IIa,b; IIc,d) in addition to isolated sub-clusters (Ie; IIe) in Fig. 6. Here it is interesting to note that in case of the eccentric sphere (Fig. 1e; Fig. 6), four distinct pairs of sub-clusters are observed to remain two-fold degenerate (i.e., sub-clusters Ia,b; Ic,d; IIa,b; IIc,d). This can be 

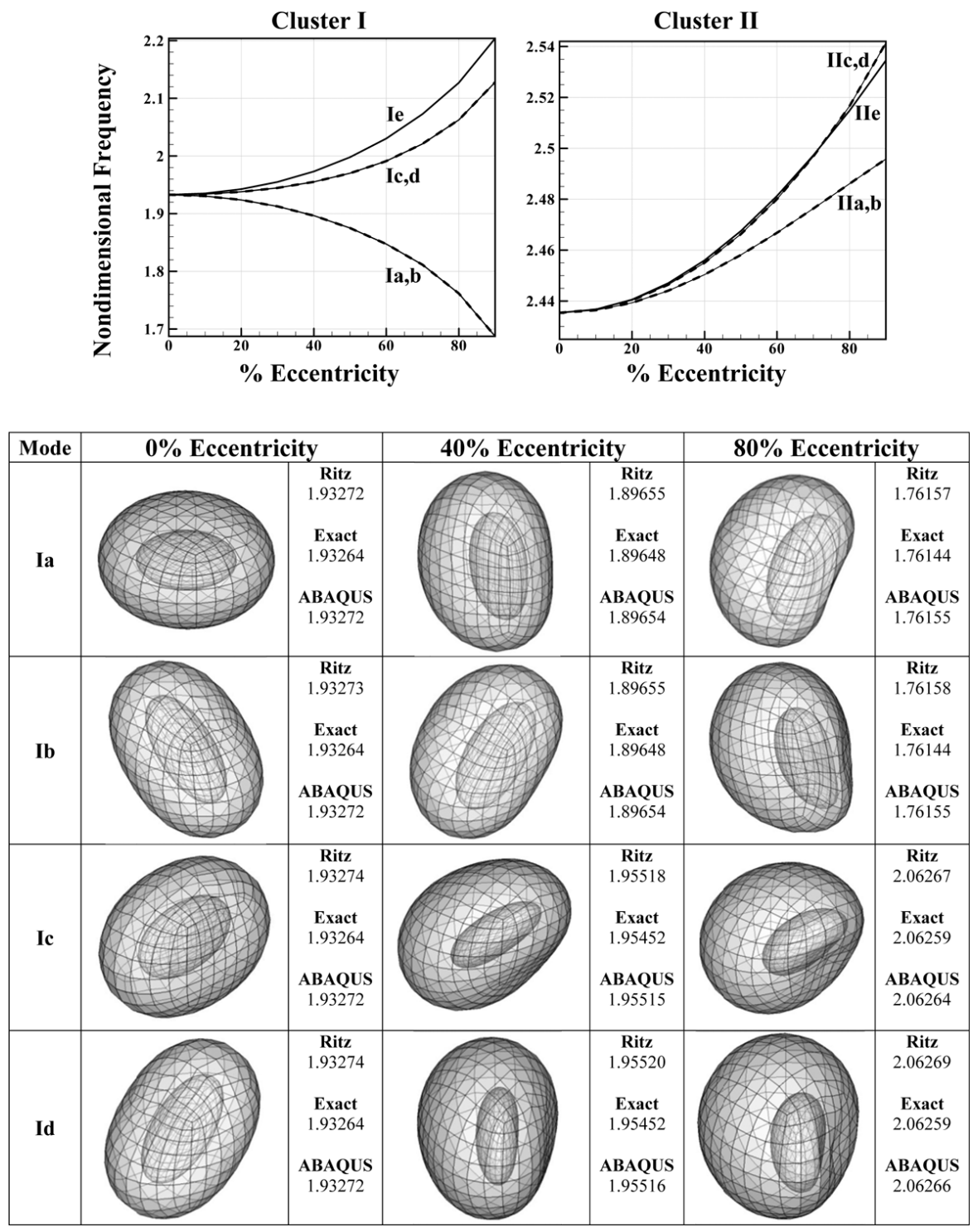

Fig. 6. Variation of the first two frequency clusters with eccentricity parameter for the solid sphere with an internal spherical cavity, along with the first four three-dimensional mode shapes for selected eccentricity parameters.

linked to preservation of some symmetry even in presence of eccentricity. Also, one should note that, as mentioned earlier, such degenerate mode pairs are exactly the same, but are merely rotated. The latter observations are to some extend valid for the eccentric cube problem (Fig. 1c) for which the cluster IIa,b remain two-fold degenerate, and their associated modes occur in pairs, regardless of cavity eccentricity (see Fig. 4).

Now, it is clear from the various frequency-eccentricity subplots that besides splitting of eigen-frequencies, the crossovers of different branches (i.e., "mode crossing" [1,2]) occur. At the crossover point, two or more modes may share the same resonance frequency. In particular, one can clearly observe: external crossovers between sub-clusters IIIa and IIb as well as between sub-clusters IIIa and IIa in Fig. 2, external crossovers between cluster II and sub-cluster Ib as well as between cluster II and sub-cluster Ia in addition to cluster III and several higher order sub-clusters in Fig. 3, external crossovers between sub-clusters IIIa and IIc as well as between sun-clusters IIIa and IIa,b in Fig. 4, internal crossover between sub-clusters IIe and IIc,d in Fig. 6. At these crossovers, corresponding to specific eccentricities, the previously split eigen-frequencies become degenerate. This implies that beyond the 
crossover eccentricity, the stiffness of structure in one vibration mode will interchange place with another (i.e., the phenomenon of "mode switching" [69,70] may occur as the cavity eccentricity varies across the cross-over points). Another interesting observation is that the frequency spectrum curves associated with the breathing mode in each case remains single-valued in the entire range of eccentricity. This can readily be observed with respect to sub-cluster IV displayed in Fig. 3 associated with the parallelepiped with an eccentric cylindrical cavity (see Fig. 1b). Here it should be noted that, as the frequency clusters associated with the breathing mode for the remaining four sub-problems (Figs 1a,c,d,e) arise at relatively high frequencies, they do not appear in the presented subplots (Figs 2 , $4,5,6)$. In other words, there is a single non-degenerate frequency associated with the breathing mode regardless of cavity eccentricity.

\section{Conclusions}

A Chebyshev-Ritz numerical procedure based on the 3D elasticity theory is employed in this work to extract the full vibration spectrum of natural frequencies along with selected3D deformed mode shapes for a class of traction-free bonded elastic solids of canonical morphology (i.e., finite cylinders, solid spheres, and rectangular parallelepipeds) weakened by presence of an eccentric spherical or cylindrical cavity. The most important observations are summarized as follows. For the elastic bodies with a perfectly concentric cavity, due to the presence of geometric symmetries, either single-valued or completely repeated fully degenerate eigen-frequencies are obtained. As eccentricity is introduced, characteristically different effects on natural frequencies are observed, depending on the problem geometry and mode type. In particular, the majority of initially observed degeneracies are lifted, and the imposed asymmetry causes some or all of the repeated vibration modes to split into modes with distinct natural frequencies. Moreover, the degeneration of remaining pairs of eigen-frequencies for a few particular geometries (e.g., the eccentric sphere and cube) is linked to preservation of some geometric symmetry even in presence of cavity eccentricity for those geometries. Also, besides splitting of eigen-frequencies, the internal and/or external crossovers (mode crossings)are frequently observed between different frequency branches. At these crossover points, corresponding to specific eccentricities, the previously split eigen-frequencies become degenerate, and two or more modes share the same resonance frequency. This implies that beyond the crossover eccentricity, the stiffness of structure in one vibration mode will interchange place with another, and the phenomenon of "mode switching" may occur as the cavity eccentricity varies across the cross-over points. Lastly, the frequency spectrum curves associated with the breathing mode for each sub-problem is observed to remain single-valued in the entire range of eccentricity (i.e., there is a single non-degenerate frequency associated with the breathing mode regardless of cavity eccentricity).

\section{Appendix}

The maximum kinetic and potential energy functions Eq. (7) may be written in a more condensed form as

$$
\begin{aligned}
& T_{\max }=\frac{\rho \omega^{2}}{2} \iiint \mathrm{K}(x, y, z) d x d y d z, \\
& V_{\max }=\frac{E}{2(1+\nu)} \iiint \mathrm{P}(x, y, z) d x d y d z
\end{aligned}
$$

where

$$
\begin{aligned}
& \mathrm{K}(x, y, z)=U^{2}(x, y, z)+V^{2}(x, y, z)+W^{2}(x, y, z), \\
& \mathrm{P}(x, y, z)=\frac{\nu}{1-2 \nu} \Gamma_{1}(x, y, z)+\frac{1}{2} \Gamma_{2}(x, y, z)+\Gamma_{3}(x, y, z),
\end{aligned}
$$

The origins of the following "un-starred" (Cartesian, Cylindrical, and Spherical) coordinate systems are positioned at the center of each elastic body: $(x, y, z),(r, \theta, z),(R, \theta, \phi)$. Similarly the "starred" coordinate systems, set at the center of each cavity, are denoted by: $\left(x^{*}, y^{*}, z^{*}\right),\left(r^{*}, \theta^{*}, z^{*}\right),\left(R^{*}, \theta^{*}, \phi^{*}\right)$. The above volume integrals are to be carried out over the domain bounded by the outer boundary of the elastic body (i.e., with respect to the "un-starred" 
coordinate system), and subsequently subtracted from the volume integral carried out over the cavity domain (i.e., with respect to the "starred" coordinate system), i.e.,

$$
T_{\max }=T_{b}^{\max }-T_{c}^{\max }, V_{\max }=V_{b}^{\max }-V_{c}^{\max },
$$

Where the subscripts " $b$ " and "c" refer to the elastic body and the cavity, respectively. Subsequently, the triple integrals will be evaluated after proper change of variables between certain coordinate systems relevant to each subproblem under consideration. Accordingly, the following standard transformations between the coordinate systems will be employed in the subsequent formulation:

Cartesian-Cartesian transformation: $x=x^{*}+e_{x}, y=y^{*}+e_{y}, z=z^{*}+e_{z}$

Cartesian-Cylindrical transformation: $x=r \cos \theta, y=r \sin \theta, z=z$

Cartesian-Spherical transformation: $x=R \sin \phi \cos \theta, y=R \sin \phi \sin \theta, z=R \cos \phi$.

Also, the proper sequence of change of coordinates implemented, and the associated volume integrals for each sub-problem (see Fig. 1) are summarized below:

Sub-problem 1: Finite cylinder with an eccentric spherical cavity (Fig. 1a)

Elastic Cylinder: $(x, y, z)$ to $(r, \theta, z)$.

Spherical cavity: $(x, y, z)$ to $\left(x^{*}, y^{*}, z^{*}\right)$, and subsequently, $\left(x^{*}, y^{*}, z^{*}\right)$ to $\left(R^{*}, \theta^{*}, \phi^{*}\right)$.

$$
\begin{aligned}
& T_{b}^{\max }=\frac{\rho \omega^{2}}{2} \int_{-L}^{L} \int_{0}^{2 \pi} \int_{0}^{b} \mathrm{~K}(r \cos \theta, r \sin \theta, z) \frac{\partial(x, y, z)}{\partial(r, \theta, z)} d r d \theta d z, \\
& T_{c}^{\max }=\frac{\rho \omega^{2}}{2} \int_{0}^{\pi} \int_{0}^{2 \pi} \int_{0}^{a} K\left(R^{*} \sin \phi^{*} \cos \theta^{*}, R^{*} \sin \phi^{*} \sin \theta^{*}+e_{y}, R^{*} \cos \phi^{*}+e_{z}\right) \frac{\partial(x, y, z)}{\partial\left(x^{*}, y^{*}, z^{*}\right)} \\
& \frac{\partial\left(x^{*}, y^{*}, z^{*}\right)}{\partial\left(R^{*}, \theta^{*}, \phi^{*}\right)} d R^{*} d \theta^{*} d \phi^{*}, \\
& V_{b}^{\max }=\frac{E}{2(1+\nu)} \int_{-L}^{L} \int_{0}^{2 \pi} \int_{0}^{b} P(r \cos \theta, r \sin \theta, z) \frac{\partial(x, y, z)}{\partial(r, \theta, z)} d r d \theta d z, \\
& V_{c}^{\max }=\frac{E}{2(1+\nu)} \int_{0}^{\pi} \int_{0}^{2 \pi} \int_{0}^{a} P\left(R^{*} \sin \phi^{*} \cos \theta^{*}, R^{*} \sin \phi^{*} \sin \theta^{*}+e_{y}, R^{*} \cos \phi^{*}+e_{z}\right) \\
& \frac{\partial(x, y, z)}{\partial\left(x^{*}, y^{*}, z^{*}\right)} \frac{\partial\left(x^{*}, y^{*}, z^{*}\right)}{\partial\left(R^{*}, \theta^{*}, \phi^{*}\right)} d R^{*} d \theta^{*} d \phi^{*},
\end{aligned}
$$

where the Jacobian determinant of the above transformations are given as:

$$
\begin{aligned}
& \frac{\partial(x, y, z)}{\partial\left(x^{*}, y^{*}, z^{*}\right)}=1, \frac{\partial(x, y, z)}{\partial(r, \theta, z)}=r, \frac{\partial(x, y, z)}{\partial(R, \theta, \phi)}=-R^{2} \sin \theta \\
& \frac{\partial\left(x^{*}, y^{*}, z^{*}\right)}{\partial\left(R^{*}, \theta^{*}, \phi^{*}\right)}=-R^{* 2} \sin \theta^{*}, \frac{\partial\left(x^{*}, y^{*}, z^{*}\right)}{\partial\left(r^{*}, \theta^{*}, z^{*}\right)}=r^{*} .
\end{aligned}
$$

Sub-problem 2: Solid parallelepiped with an eccentric cylindrical cavity (Fig. 1b)

Elastic parallelepiped: $(x, y, z)$.

Spherical cavity: $(x, y, z)$ to $\left(x^{*}, y^{*}, z^{*}\right)$, and subsequently, $\left(x^{*}, y^{*}, z^{*}\right)$ to $\left(r^{*}, \theta^{*}, z^{*}\right)$. 


$$
\begin{aligned}
& T_{b}^{\max }=\frac{\rho \omega^{2}}{2} \int_{-L}^{L} \int_{-c}^{c} \int_{-b}^{b} K(x, y, z) d x d y d z, \\
& T_{c}^{\max }=\frac{\rho \omega^{2}}{2} \int_{-L}^{L} \int_{0}^{2 \pi} \int_{0}^{a} K\left(r^{*} \cos \theta^{*}+e_{x}, r^{*} \sin \theta^{*}+e_{y}, z^{*}\right) \frac{\partial(x, y, z)}{\partial\left(x^{*}, y^{*}, z^{*}\right)} \frac{\partial\left(x^{*}, y^{*}, z^{*}\right)}{\partial\left(r^{*}, \theta^{*}, z^{*}\right)} d r^{*} d \theta^{*} d z^{*}, \\
& V_{b}^{\max }=\frac{E}{2(1+\nu)} \int_{-L}^{L} \int_{-c}^{c} \int_{-b}^{b} P(x, y, z) d x d y d z, \\
& V_{c}^{\max }=\frac{E}{2(1+\nu)} \int_{-L}^{L} \int_{0}^{2 \pi} \int_{0}^{a} P\left(r^{*} \cos \theta^{*}+e_{x}, r^{*} \sin \theta^{*}+e_{y}, z^{*}\right) \frac{\partial(x, y, z)}{\partial\left(x^{*}, y^{*}, z^{*}\right)} \frac{\partial\left(x^{*}, y^{*}, z^{*}\right)}{\partial\left(r^{*}, \theta^{*}, z^{*}\right)} d r^{*} d \theta^{*} d z^{*} .
\end{aligned}
$$

Sub-problem 3: Solid parallelpiped with an eccentric spherical cavity (Fig. 1c)

Elastic parallelepiped: $(x, y, z)$.

Spherical cavity: $(x, y, z)$ to $\left(x^{*}, y^{*}, z^{*}\right)$, and subsequently, $\left(x^{*}, y^{*}, z^{*}\right)$ to $\left(R^{*}, \theta^{*}, \phi^{*}\right)$.

$$
\begin{aligned}
T_{b}^{\max } & =\frac{\rho \omega^{2}}{2} \int_{-L}^{L} \int_{-c}^{c} \int_{-b}^{b} K(x, y, z) d x d y d z \\
T_{c}^{\max } & =\frac{\rho \omega^{2}}{2} \int_{0}^{\pi} \int_{0}^{2 \pi} \int_{0}^{a} K\left(R^{*} \sin \phi^{*} \cos \theta^{*}+e_{x}, R^{*} \sin \phi^{*} \sin \theta^{*}+e_{y}, R^{*} \cos \phi^{*}+e_{z}\right) \\
\frac{\partial(x, y, z)}{\partial\left(x^{*}, y^{*}, z^{*}\right)} & \frac{\partial\left(x^{*}, y^{*}, z^{*}\right)}{\partial\left(R^{*}, \theta^{*}, \phi^{*}\right)} d R^{*} d \theta^{*} d \phi^{*}, \\
V_{b}^{\max } & =\frac{E}{2(1+\nu)} \int_{-L}^{L} \int_{-c}^{c} \int_{-b}^{b} P(x, y, z) d x d y d z, \\
V_{c}^{\max } & =\frac{E}{2(1+\nu)} \int_{0}^{\pi} \int_{0}^{2 \pi} \int_{0}^{a} P\left(R^{*} \sin \phi^{*} \cos \theta^{*}+e_{x}, R^{*} \sin \phi^{*} \sin \theta^{*}+e_{y}, R^{*} \cos \phi^{*}+e_{z}\right) \\
\frac{\partial(x, y, z)}{\partial\left(x^{*}, y^{*}, z^{*}\right)} & \frac{\partial\left(x^{*}, y^{*}, z^{*}\right)}{\partial\left(R^{*}, \theta^{*}, \phi^{*}\right)} d R^{*} d \theta^{*} d \phi^{*} .
\end{aligned}
$$

Sub-problem 4: Finite cylinder with an eccentric cylindrical cavity (Fig. 1d)

Elastic Cylinder: $(x, y, z)$ to $(r, \theta, z)$.

Spherical cavity: $(x, y, z)$ to $\left(x^{*}, y^{*}, z^{*}\right)$, and subsequently, $\left(x^{*}, y^{*}, z^{*}\right)$ to $\left(r^{*}, \theta^{*}, z^{*}\right)$.

$$
T_{b}^{\max }=\frac{\rho \omega^{2}}{2} \int_{-L}^{L} \int_{0}^{2 \pi} \int_{0}^{b} K(r \cos \theta, r \sin \theta, z) \frac{\partial(x, y, z)}{\partial(r, \theta, z)} d r d \theta d z,
$$




$$
\begin{aligned}
T_{c}^{\max } & =\frac{\rho \omega^{2}}{2} \int_{-L}^{L} \int_{0}^{2 \pi} \int_{0}^{a} K\left(r^{*} \cos \theta^{*}, r^{*} \sin \theta^{*}+e_{y}, z^{*}\right) \frac{\partial(x, y, z)}{\partial\left(x^{*}, y^{*}, z^{*}\right)} \frac{\partial\left(x^{*}, y^{*}, z^{*}\right)}{\partial\left(r^{*}, \theta^{*}, z^{*}\right)} d r^{*} d \theta^{*} d z^{*}, \\
V_{b}^{\max } & =\frac{E}{2(1+\nu)} \int_{-L}^{L} \int_{0}^{2 \pi} \int_{0}^{b} P(r \cos \theta, r \sin \theta, z) \frac{\partial(x, y, z)}{\partial(r, \theta, z)} d r d \theta d z, \\
V_{c}^{\max } & =\frac{E}{2(1+\nu)} \int_{-L}^{L} \int_{0}^{2 \pi} \int_{0}^{a} P\left(r^{*} \cos \theta^{*}, r^{*} \sin \theta^{*}+e_{y}, z^{*}\right) \frac{\partial(x, y, z)}{\partial\left(x^{*}, y^{*}, z^{*}\right)} \frac{\partial\left(x^{*}, y^{*}, z^{*}\right)}{\partial\left(r^{*}, \theta^{*}, z^{*}\right)} d r^{*} d \theta^{*} d z^{*}
\end{aligned}
$$

Sub-problem 5: Solid sphere with an eccentric spherical cavity (Fig. 1e)

Elastic Cylinder: $(x, y, z)$ to $(R, \theta, \phi)$.

Spherical cavity: $(x, y, z)$ to $\left(x^{*}, y^{*}, z^{*}\right)$, and subsequently, $\left(x^{*}, y^{*}, z^{*}\right)$ to $\left(r^{*}, \theta^{*}, z^{*}\right)$.

$$
\begin{aligned}
& T_{b}^{\max }=\frac{\rho \omega^{2}}{2} \int_{0}^{\pi} \int_{0}^{2 \pi} \int_{0}^{b} K(R \sin \phi \cos \theta, R \sin \phi \sin \theta, R \cos \phi) \frac{\partial(x, y, z)}{\partial(R, \theta, \phi)} d R d \theta d \phi \\
& T_{c}^{\max }=\frac{\rho \omega^{2}}{2} \int_{0}^{\pi} \int_{0}^{2 \pi} \int_{0}^{a} K\left(R^{*} \sin \phi^{*} \cos \theta^{*}, R^{*} \sin \phi^{*} \sin \theta^{*}+e_{y}, R^{*} \cos \phi^{*}\right) \frac{\partial(x, y, z)}{\partial\left(x^{*}, y^{*}, z^{*}\right)} \\
& \frac{\partial\left(x^{*}, y^{*}, z^{*}\right)}{\partial\left(R^{*}, \theta^{*}, \phi^{*}\right)} d R^{*} d \theta^{*} d \phi^{*}, \\
& V_{b}^{\max }=\frac{E}{2(1+\nu)} \int_{0}^{\pi} \int_{0}^{2 \pi} \int_{0}^{b} P(R \sin \phi \cos \theta, R \sin \phi \sin \theta, R \cos \phi) \frac{\partial(x, y, z)}{\partial(R, \theta, \phi)} d R d \theta d \phi, \\
& V_{c}^{\max }=\frac{E}{2(1+\nu)} \int_{0}^{\pi} \int_{0}^{2 \pi} \int_{0}^{a} P\left(R^{*} \sin \phi^{*} \cos \theta^{*}, R^{*} \sin \phi^{*} \sin \theta^{*}+e_{y}, R^{*} \cos \phi^{*}\right) \\
& \frac{\partial(x, y, z)}{\partial\left(x^{*}, y^{*}, z^{*}\right)} \frac{\partial\left(x^{*}, y^{*}, z^{*}\right)}{\partial\left(R^{*}, \theta^{*}, \phi^{*}\right)} d R^{*} d \theta^{*} d \phi^{*} .
\end{aligned}
$$

\section{References}

[1] S.M. Hasheminejad and Y. Mirzaei, Exact 3D elasticity solution for free vibrations of an eccentric hollow sphere, Journal of Sound and Vibration 330 (2011), 229-244.

[2] S.M. Hasheminejad and Y. Mirzaei, Free vibration analysis of an eccentric hollow cylinder using exact 3D elasticity theory, Journal of Sound and Vibration 326 (2009), 687-702.

[3] G.R. Buchanan and C.B.Y. Yii, Effect of symmetrical boundary conditions on the vibration of thick hollow cylinders, Applied Acoustics 63 (2002), 547-566.

[4] J.P. Agnantiaris, D. Polyzos and D.E. Beskos, Free vibration analysis of non-axisymmetric and axisymmetric structures by the dual reciprocity BEM, Engineering Analysis with Boundary Elements 25 (2001), 713-723.

[5] D. Zhou, Y.K. Cheung and S.H. Lo, Three-dimensional vibration analysis of toroidal sectors with solid circular cross-sections, Journal of Applied Mechanics Transactions ASME 77 (2010), 1-8.

[6] S. Bashmal, R. Bhat and S. Rakheja, In-plane free vibration analysis of an annular disk with point elastic support, Shock and Vibration 18 (2011), 627-640.

[7] B. Uymaz and M. Aydogdu, Three-Dimensional Vibration Analysis of Functionally Graded Plates Under Various Boundary Conditions, Journal of Reinforced Plastics and Composites 26 (2007), 1847-1863. 
[8] A.W. Leiss and J. So, Accurate vibration frequencies of circular cylinders from three-dimensional analysis, Journal of the Acoustical Society of America 98 (1995), 2136-2141.

[9] A.W. Leiss and J. So, Free vibrations of thick hollow circular cylinders from three-dimensional analysis, Journal of Vibration and Acoustics Transactions of the ASME 119 (1997), 89-95.

[10] A.W. Leissa and J. So, Three-dimensional vibrations of thick circular and annular plates, Journal of Sound and Vibration 209 (1998), $15-41$.

[11] K.M. Liew and B. Yang, Three-dimensional elasticity solutions for free vibrations of circular plates: A polynomials-Ritz analysis, Computer Methods in Applied Mechanics and Engineering 175 (1999), 189-201.

[12] K.M. Liew and B. Yang, Elasticity solutions for free vibrations of annular plates from three-dimensional analysis, International Journal of Solids and Structures 37 (2000), 7689-7702.

[13] K.M. Liew, K.C. Hung and M.K. Lim, Modeling three-dimensional vibration of elliptic bars, Journal of the Acoustical Society of America 98 (1995), 1518-1526.

[14] K.M. Liew, K.C. Hung and M.K. Lim, Vibration of stress-free hollow cylinders of arbitrary cross section, Journal of Applied Mechanics 62 (1995), 718-724.

[15] D. Zhou, Y.K. Cheung, S.H. Lo and F.T.K. Au, 3D vibration analysis of rectangular plates with mixed boundary conditions, ASME Journal of Applied Mechanics 72 (2005), 227-236.

[16] D. Zhou, F.T.K. Au, Y.K. Cheung and S.H. Lo, Effect of built-in edges on 3-D vibrational characteristics of thick circular plates, International Journal of Solids and Structures 43 (2006), 1960-1978.

[17] D. Zhou, S.H. Lo, F.T.K. Au and Y.K. Cheung, Three-dimensional free vibration of thick circular plates on Pasternak foundation, Journal of Sound and Vibration 292 (2006), 726-741.

[18] D. Zhou, Y.K. Cheung, S.H. Lo and F.T.K. Au, 3D vibration analysis of solid and hollow circular cylinders via Chebyshev-Ritz method, Computer Methods in Applied Mechanics and Engineering 192 (2003), 1575-1589.

[19] A. Leissa and Zd. Zhang, On the three-dimensional vibrations of the cantilevered rectangular parallelepiped, Journal of the Acoustical Society of America 73 (1983), 2013-2021.

[20] A.W. Leissa and K.I. Jacob, Three-dimensional vibrations of twisted cantilevered parallelepipeds, Journal of Applied Mechanics Transactions ASME $\mathbf{5 3}$ (1986), 614-618.

[21] T. Irie, G. Yamada and Y. Tagawa, Three-dimensional vibration analysis of a truncated quadrangular pyramid, Journal of Applied Mechanics Transactions ASME 54 (1987), 115-120.

[22] Y. Narita, Dimensional vibration analysis of arbitrarily shaped elastic bodies by plane approximation, Nippon Kikai Gakkai Ronbunshu, C Hen/Transactions of the Japan Society of Mechanical Engineers Part C 56 (1990), 1125-1129.

[23] O.G. McGee and A.W. Leissa, Three-dimensional free vibrations of thick skewed cantilevered plates, Journal of Sound and Vibration 44 (1991), 305-322.

[24] P.G. Young and S.M. Dickinson, Free vibration of a class of solids with cavities, International Journal of Mechanical Sciences 36 (1994), 1099-1107.

[25] O.G. McGee and S.C. Spry, A three-dimensional analysis of the spheroidal and toroidal elastic vibrations of thick-walled spherical bodies of revolution, International Journal for Numerical Methods in Engineering 40 (1997), 1359-1382.

[26] J.H. Kang and A.W. Leissa, Three-dimensional vibrations of thick circular rings with isosceles trapezoidal and triangular cross-section, Journal of Vibration and Acoustics Transactions of the ASME 122 (2000), 132-139.

[27] A.V. Singh and L. Subramaniam, Vibration of thick circular disks and shells of revolution, Journal of Applied Mechanics, Transactions ASME 71 (2003), 292-298.

[28] J.H. Kang, Three-dimensional vibration analysis of thick, circular and annular plates with nonlinear thickness variation, Computers and Structures 81 (2003), 1663-1675.

[29] J. Kang and A.W. Leissa, Three-dimensional vibrations of solid cones with and without an axial circular cylindrical hole, International Journal of Solids and Structures 41 (2004), 3735-3746.

[30] H.J. Shim and J.H. Kang, Free vibrations of solid and hollow hemi-ellipsoids of revolution from a three-dimensional theory, International Journal of Engineering Science 42 (2004) 1793-1815.

[31] J. Kang and A.W. Leissa, Free vibration analysis of complete paraboloidal shells of revolution with variable thickness and solid paraboloids from a three-dimensional theory, Computers and Structures 83 (2005), 2594-2608.

[32] O.G. McGee and J.W. Kim, Three-dimensional vibrations of cylindrical elastic solids with V-notches and sharp radial cracks, Journal of Sound and Vibration 329 (2010), 457-484.

[33] K.M. Liew, K.C. Hung and M.K. Lim, A continuum three-dimensional vibration analysis of thick rectangular plates, International Journal of Solids and Structures 30 (1993), 3357-3379.

[34] K.M. Liew, K.C. Hung and M.K. Lim, Three-dimensional elasticity solutions to vibration of cantilevered skewed trapezoids, AIAA journal 32 (1994), 2080-2089.

[35] K.C. Hung, K.M. Liew and M.K. Lira, Free vibration of cantilevered cylinders: effects of cross-sections and cavities, Acta Mechanica 113 (1995), 37-52.

[36] C.W. Lim, K.M. Liew and S. Kitipornchai, Vibration of open cylindrical shells: A three-dimensional elasticity approach, Journal of the Acoustical Society of America 104 (1998), 1436-1443.

[37] H. Nagino, T. Mikami and T. Mizusawa, Three-dimensional free vibration analysis of isotropic rectangular plates using the B-spline Ritz method, Journal of Sound and Vibration 317 (2008), 329-353.

[38] D. Zhou, Y.K. Cheung, F.T.K. Au and S.H. Lo, Three-dimensional vibration analysis of thick rectangular plates using Chebyshev polynomial and Ritz method, International Journal of Solids and Structures 39 (2002), 6339-6353.

[39] D. Zhou, F.T.K. Au, S.H. Lo and Y.K. Cheung, Three-dimensional vibration analysis of a torus with circular cross section, Journal of the Acoustical Society of America 112 (2002), 2831-2839. 
[40] D. Zhou, F.T.K. Au, Y.K. Cheung and S.H. Lo, Three-dimensional vibration analysis of circular and annular plates via the Chebyshev Ritz method, International Journal of Solids and Structures 40 (2003), 3089-3105.

[41] D. Zhou, Y.K. Cheung, S.H. Lo and F.T.K. Au, Three-dimensional vibration analysis of rectangular thick plates on Pasternak foundation, International Journal for Numerical Methods in Engineering 59 (2004), 1313-1334.

[42] D. Zhou, S.H. Lo, Y.K. Cheung and F.T.K. Au, 3-D vibration analysis of generalized super elliptical plates using Chebyshev-Ritz method, International Journal of Solids and Structures 41 (2004), 4697-4712.

[43] D. Zhou, W. Liu and Q. Yang, Three-dimensional vibration analysis of cantilevered skew plates, Journal of Sound and Vibration 313 (2008), 134-148.

[44] M. Kim, J. Moon and J.A. Wickert, Spatial modulation of repeated vibration modes in rotationally periodic structures, Journal of Vibration and Acoustics Transactions of the ASME 122 (2000), 62-68.

[45] R.C. Yu and Jr.C.D. Mote, Vibration and parametric excitation in asymmetric circular plates under moving loads, Journal of Sound and Vibration 119 (1987), 409-427.

[46] Z.L. Chen and S.Y. Zhou, The chaotic attractor of the precursory field of the seimogenic system and its fractal dimension, Acta Seismologica Sinica 6 (1993), 913-921.

[47] I.Y. Shen and J.C.D. Mote, On the mode splitting of degenerate mechanical systems containing cracks, Journal of Applied Mechanics Transactions ASME 60 (1993), 929-934.

[48] J.G. Tseng and J.A. Wickert, Vibration of an eccentrically clamped annular plate, Journal of Vibration and Acoustics Transactions of the ASME 116 (1994), 155-160.

[49] R.G. Parker and C.D. Mote, Jr., Exact perturbation for the vibration of almost annular or circular plates, Journal of Vibration and Acoustics Transactions of the ASME 118 (1996), 436-445.

[50] R.G. Parker and C.D. Mote, Jr., Vibration and coupling phenomena in asymmetric disk-spindle systems, Journal of Applied Mechanics, Transactions ASME 62 (1996), 953-961.

[51] R.G. Parker and C.D. Mote, Jr., Exact boundary condition perturbation for eigensolutions of the wave equation, Journal of Sound and Vibration 211 (1998), 389-407.

[52] J.Y. Chang and J.A. Wickert, Measurement and analysis of modulated doublet mode response in mock bladed disks, Journal of Sound and Vibration 250(2001), 379-400.

[53] M. H. Amini, M. Soleimani and A. Rastgoo, Three-dimensional free vibration analysis of functionally graded material plates resting on an elastic foundation, Smart Materials and Structures 18 (2009), 085015 (9pp).

[54] Y.Z. Chen, Stress analysis of a cylindrical bar with a spherical cavity or rigid inclusion by the eigenfunction expansion variational method, International Journal of Engineering Science 42 (2004), 325-338.

[55] H.F. Tiersten and D.V. Shick, Resonant wave propagation in gun tubes with eccentric bores, Wave Motion 21 (1995), $75-84$.

[56] E. Tsuchida, K. Kawashima and I. Nakahara, Stresses in an elastic circular cylinder with a spherical cavity under transverse bending, Bull JSME 19 (1976), 1-7.

[57] E. Tsuchida and I. Nakahara, Three-dimensional stress concentration around a spherical cavity in thick plate under uniaxial tension, Bull JSME 19 (1976), 1107-1114.

[58] L.G. Smirnov and I.I. Fedik, Elastic stresses in a sphere with an exogenous eccentric spherical inclusion, Journal of Soviet Mathematics 64 (1993), 966-970.

[59] M. Hamada and J. Kodama, Tension of a circular cylinder containing spherical cavity, Bulletin of the JSME 28 (1985), 776-783.

[60] B.R. Tittmann, Scattering of elastic waves from simple defects in solids, a review, Wave Motion 5 (1983), $299-306$.

[61] J. Jellison, H.R. Kess, D.E. Adams and D.C. Nelson, Vibration-based NDE technique for identifying non-uniformities in manufacture parts with degeneracies, in Proceedings of IMECE, New Orleans, LA, 2002, 621-628.

[62] M. de Billy and A.C. Hladky-Hennion, On the splitting of aspherical structure submitted to an acoustic polarized excitation, Ultrasonics 43 (2004), 27-34.

[63] J.G. Minonzio, F.D. Philippe, C. Prada and M. Fink, Characterization of an elastic cylinder and an elastic sphere with the time-reversal operator: application to the sub-resolution limit, Inverse Problems 24 (2008), art. no. 025014 (24pp).

[64] A.O. Vatul'yan and O.A. Belyak, Various methods of reconstruction of a cavity in an orthotropic layer, Journal of Applied Mechanics and Technical Physics $\mathbf{5 0}$ (2009), 512-518.

[65] C.B. Ling and C.P. Tsai, Stresses in a thick plate containing an eccentric spherical inclusion or cavity Part I, Acta Mechanica 7 (1969), $169-186$.

[66] K. Baganas, A. Charalambopoulos and G.D. Manolis, Detection of spherical inclusions in a bounded, elastic cylindrical domain, Wave Motion 41 (2005), 13-28.

[67] S. Wolfram, Mathematica: A System for Doing Mathematics by Computer. second ed., Addison Wesley Publishing Company, Reading, MA, 1991.

[68] ABAQUS, Analysis User's Manual Version 6.6 On-line Documentation.

[69] Y. Xiang and L. Zhang, Free vibration analysis of stepped circular Mindlin plates, Journal of Sound and Vibration 280 (2005), $633-655$.

[70] C.F. Lu, Z.Y. Huang and W.Q. Chen, Semi-analytical solutions for free vibration of anisotropic laminated plates in cylindrical bending, Journal of Sound and Vibration 304 (2007), 987-995. 

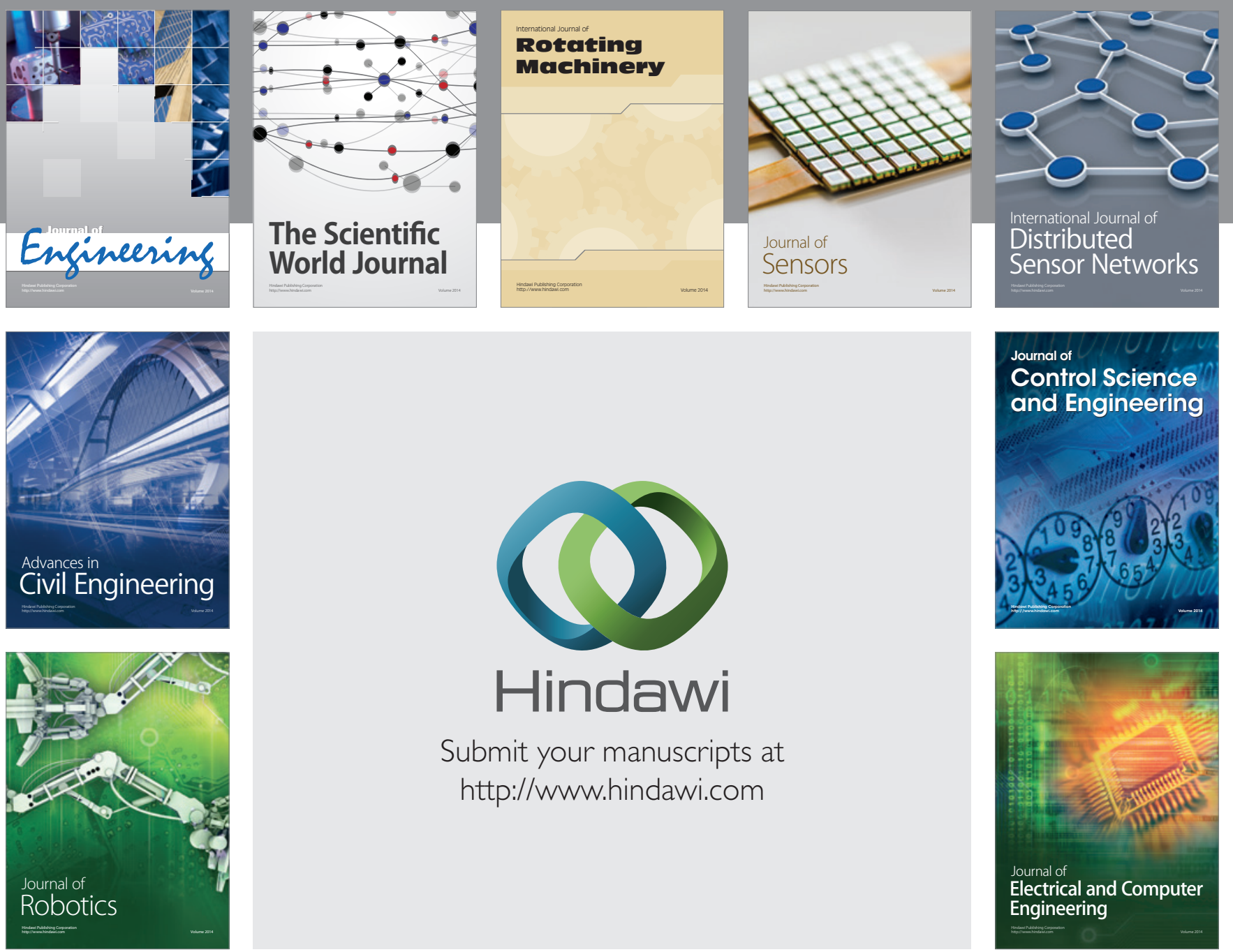

Submit your manuscripts at

http://www.hindawi.com
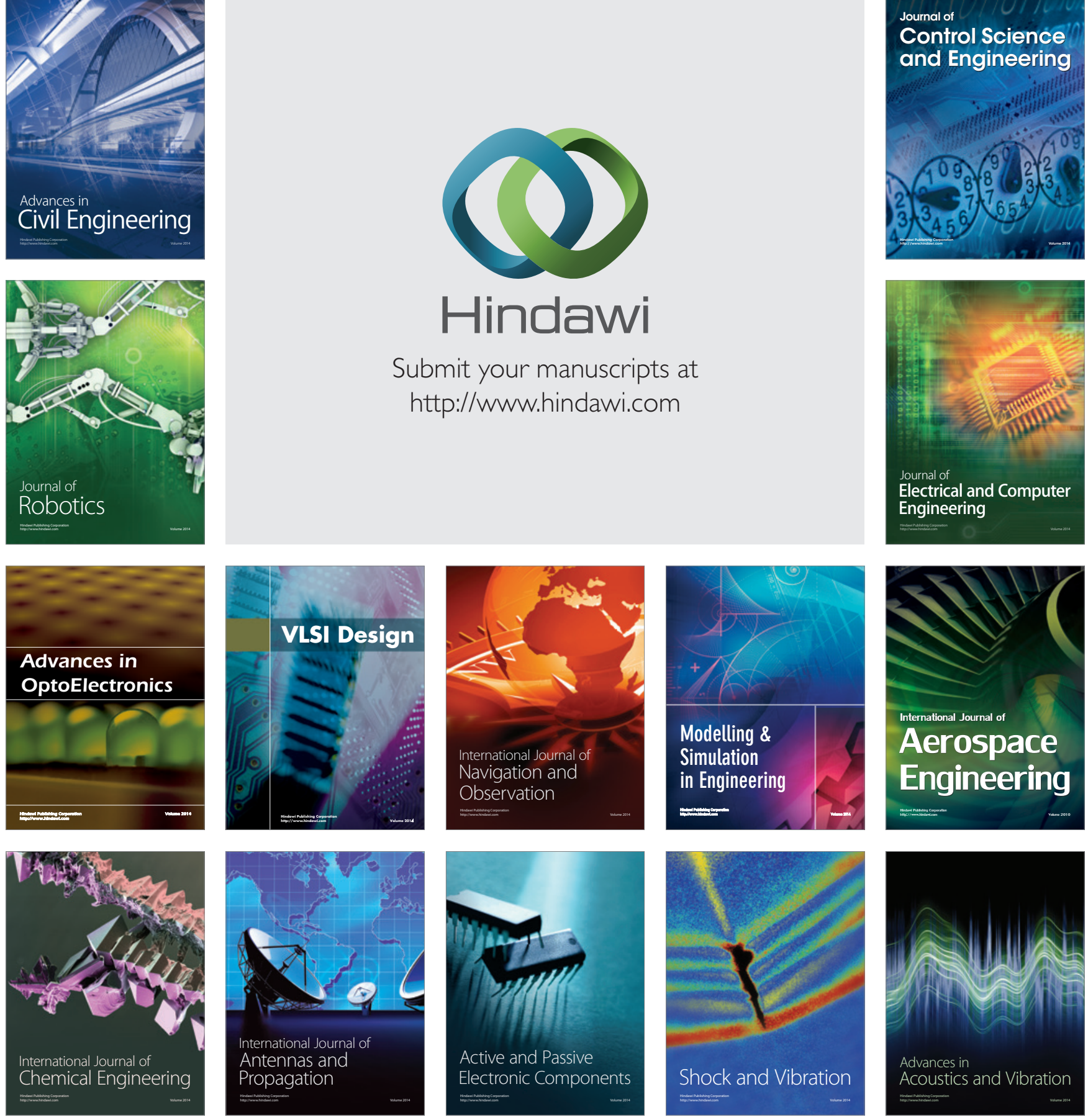